\title{
Target-site resistance to neonicotinoids
}

\author{
Andrew J. Crossthwaite • Stefano Rendine • \\ Marco Stenta $\cdot$ Russell Slater
}

Received: 2 April 2014 / Accepted: 29 June 2014 / Published online: 17 July 2014

(C) Springer-Verlag Berlin Heidelberg 2014

\begin{abstract}
Neonicotinoid insecticides selectively target the invertebrate nicotinic acetylcholine receptor and disrupt excitatory cholinergic neurotransmission. First launched over 20 years ago, their broad pest spectrum, variety of application methods and relatively low risk to nontarget organisms have resulted in this class dominating the insecticide market with global annual sales in excess of $\$ 3.5 \mathrm{bn}$. This remarkable commercial success brings with it conditions in the field that favour selection of resistant phenotypes. A number of important pest species have been identified with mutations at the nicotinic acetylcholine receptor associated with insensitivity to neonicotinoids. The detailed characterization of these mutations has facilitated a greater understanding of the invertebrate nicotinic acetylcholine receptor.
\end{abstract}

Keywords Neonicotinoid · Nicotinic $\cdot$ Resistance $\cdot$ Target $\cdot$ Site

The nicotinic acetylcholine receptor (nAChR) is a ligandgated ion channel responsible for mediating excitatory cholinergic neurotransmission in the central nervous system of invertebrates and is the target site for the neonicotinoid

Electronic supplementary material The online version of this article (doi:10.1007/s12154-014-0116-y) contains supplementary material, which is available to authorized users.

A. J. Crossthwaite $(\bowtie)$

Biological Sciences, Jealott's Hill International Research Centre, Syngenta Crop Protection, Bracknell RG42 6EY, UK

e-mail: andrew.crossthwaite@syngenta.com

S. Rendine $\cdot$ M. Stenta

Insecticide Chemistry, Werk Stein, Syngenta Crop Protection, CH4332 Schaffhauserstrasse, Stein, Switzerland

R. Slater

Biological Sciences, Werk Stein, Syngenta Crop Protection, CH4332 Schaffhauserstrasse, Stein, Switzerland insecticides. The neonicotinoids, along with spinosyns and nereistoxin analogues, are the three principal classes of insecticides that mediate their effect by disrupting the normal physiological workings of the nAChR [1]. Of these, the systemically delivered neonicotinoids are by far the most commercially successful, with annual global sales in excess of $\$ 3.7$ billion [2]. First introduced to the market in 1991, the neonicotinoids rapidly established themselves over older chemistry classes such as organophosphates and carbamates, due to their favourable safety profile, wide pest spectrum and multiple application methods. In total, there are now eight insecticides commercialized with a neonicotinoid mode of action, with further examples in development [1, 3]. Thiamethoxam and imidacloprid dominate the market, accounting for approximately two-thirds of total sales [4]. Neonicotinoids are highly potent on hemipteran sap-feeding insects (e.g. aphids, whiteflies, hoppers, scales, etc.) but also used to control a range of foliar-feeding insects (e.g. Colorado potato beetle, etc.) and via seed treatment a range of soil pests (e.g. corn rootworms, white grubs, wireworms, etc.) [4].

The nAChR is composed of a hetero or homo-pentamer subunit combination organized around a central cation selective pore. There are two orthosteric binding sites at each $\mathrm{nAChR}$ heteropentamer, occurring at the extracellular domain at the interface between adjacent $\alpha$ and $\beta$ subunits, donating loops $\mathrm{A}$ to $\mathrm{C}$ and loops $\mathrm{D}$ to $\mathrm{E}$, respectively, for the interaction with the substrate [5]. Within the binding pocket, neonicotinoids stack against tyrosine residues from loops $\mathrm{A}$ and $\mathrm{C}$ and tryptophan residues from loops $\mathrm{B}$ and $\mathrm{D}$ [6]. The backbone of loop E, possibly through water-mediated contacts, and the arginine on loop D provide further spots essential for ligand recognition through polar interactions.

The sequencing of insect genomes has revealed that insects have $\sim 10$ different $n A C h R$ subunit genes [7]. It is highly likely that, as in mammals, different combinations of subunits result in unique receptor subtypes with differing pharmacology. 
Indeed, invertebrate nAChRs can be broadly separated into two classes based on their differing sensitivity to $\alpha$ bungarotoxin and neonicotinoids [8]. However, the precise pentameric subunit stoichiometry underlying this differential sensitivity is unknown. An interesting feature of imidacloprid pharmacology in hemipteran insects is the presence of dual affinity binding sites. Aphids and hoppers have both a very high affinity site (pM) and a secondary lower affinity (nM) site to imidacloprid $[9,10]$. However, in the majority of other insects studied, only a single site of approximately nanomolar affinity is thought to be present [9]. The unique presence of a very high affinity binding site in hemipteran insects has been suggested to underlie the exquisite sensitivity of this insect class to the neonicotinoids. The subunit stoichiometry underlying the different affinity sites in hemipteran insects is not fully clear and unlike mammalian nAChRs, there has been no successful in-vitro expression of a functional insect $\mathrm{nAChR}$ heteropentamer due to the challenges of expressing the $\beta$ subunit [11]. Despite this, the nAChR has been one of the most intensively studied invertebrate receptors and much progress has been made on understanding its properties. Contributing to this has been the discovery and detailed genetic, pharmacological and modelling characterization of two independent examples of target-site resistance to the neonicotinoids $[12,13]$. Although neonicotinoids are one of the most commercially successful classes of insecticide, target-site resistance to them has been relatively slow to develop and the majority of resistance to date has been attributed to enhanced metabolism by a variety of detoxification enzymes. At the time of writing, there are currently 13 species of agricultural pests that are documented as being resistant to the $\mathrm{nAChR}$ agonist class of insecticides, which includes the neonicotinoids. The over-expression of detoxifying P450 monoxygenases has been strongly associated with neonicotinoid resistance in Bemisia tabaci [14-16], Trialeurodes vaporariorum [17], Nilaparvata lugens [18], Myzus persicae [19] and Leptinotarsa decemlineata [20], whilst elevated expression levels of esterases and glutathione-S-transferases have been associated with cases of resistance to Amrasca biguttula [21], Diaphorina citri [22] and Aphis gossypii [23]. Although in general cross-resistance is observed across all insecticides in the neonicotinoid class, the level of resistance can be variable between individual compounds and species. However, there are a small number of hemipteran species in which resistance has been confirmed to be associated with a target-site resistance. Selection pressure on $N$. lugens with imidacloprid over 35 generations under laboratory conditions resulted in a 250 -fold level of resistance [13]. Subsequent cloning of $\mathrm{nAChR} \mathrm{Nl} \alpha$ subunits revealed several polymorphisms but only one associated with highlevel imidacloprid resistance, the replacement of tyrosine at position 151 within loop B to a serine in both $\mathrm{Nl} \alpha 1$ and $\mathrm{Nl} \alpha 3$. It is of interest to note that homozygotes with mutations in both $\mathrm{Nl} \alpha$ subunits were required for very-high-level resistance. Heterozygous $N$. lugens displayed a lower level of resistance, suggesting that the Y151S mutation is required in two separate $\mathrm{nAChR}$ genes for high-level resistance to occur [13]. The presence of the Y151S mutation did not affect the total $\mathrm{nAChR}$ receptor expression levels, either in membranes from $N$. lugens or when expressed heterologously in cells (co-expressed with rat $\beta$ subunits). However, Y151S significantly reduces the affinity of $\left[{ }^{3} \mathrm{H}\right]$-IMD binding, as well as reducing imidacloprid agonist activity in co-expressing systems [24-26]. Despite the level of insensitivity to neonicotinoids, the Y151S mutation has never been observed in the field, and this is likely to be due to the high fitness penalty associated with this amino acid change [27]. The Y151 residue is not thought to underlie the selectivity of neonicotinoids to invertebrates since the residue is highly conserved in mammals; rather, the effect of the mutation is more subtle. Since Y151 is not in close contact with the neonicotinoid binding site, its mutation has an indirect effect on ligand interaction, which is best revealed by an extended molecular dynamics simulation, capable of capturing subtle differences in the local disorder of the protein structure. The molluscan AChBP is homologous to the extracellular Nterminal region of nAChRs, and co-crystalization studies in the presence of neonicotinoids have provided deep insights into the molecular interactions involved in binding. In order to highlight the energetic and structural features underlying the Y151 target-site resistance to neonicotinoids, in this study, we performed molecular dynamics simulations [28] on imidacloprid in complex with $M$. persicae nAChR (for ease of simulation, $M$. persicae was chosen as a surrogate representative for hemipteran insects) and the $\alpha 2 \beta 1$ subunit stoichiometry was adopted [6]. Homology models of the wildtype and mutated receptor were used (details on the model construction and simulation setup are available in the Supplementary information material). Mutation of Y176 in the M. persicae model (corresponding to Y151 in N. lugens by sequence alignment) into serine resulted in an increased flexibility of loop B of the $\alpha$ subunit. The mutation particularly affected the mobility of W174, which is known to have a crucial role in neonicotinoid binding (Fig. 1a) [29]. Quantitatively, the values of the backbone and sidechain dihedral angles of W174 (Fig. 1b, see Supplementary information), monitored throughout the simulations, were consistently found to have higher standard deviations in the Y176S system, as compared to the wild type (Fig. 1c and Table S1 Supplementary information). This finding indicates that, in the mutated system, W174 has a reduced probability to be optimally oriented for the interaction with the substrate, resulting in a decreased sensitivity to neonicotinoids.

The first field evolved nAChR mutations associated with high level imidacloprid resistance was identified in aphids. A strain of $M$. persicae, clone $\mathrm{FRC,} \mathrm{isolated} \mathrm{from} \mathrm{peach}$ 

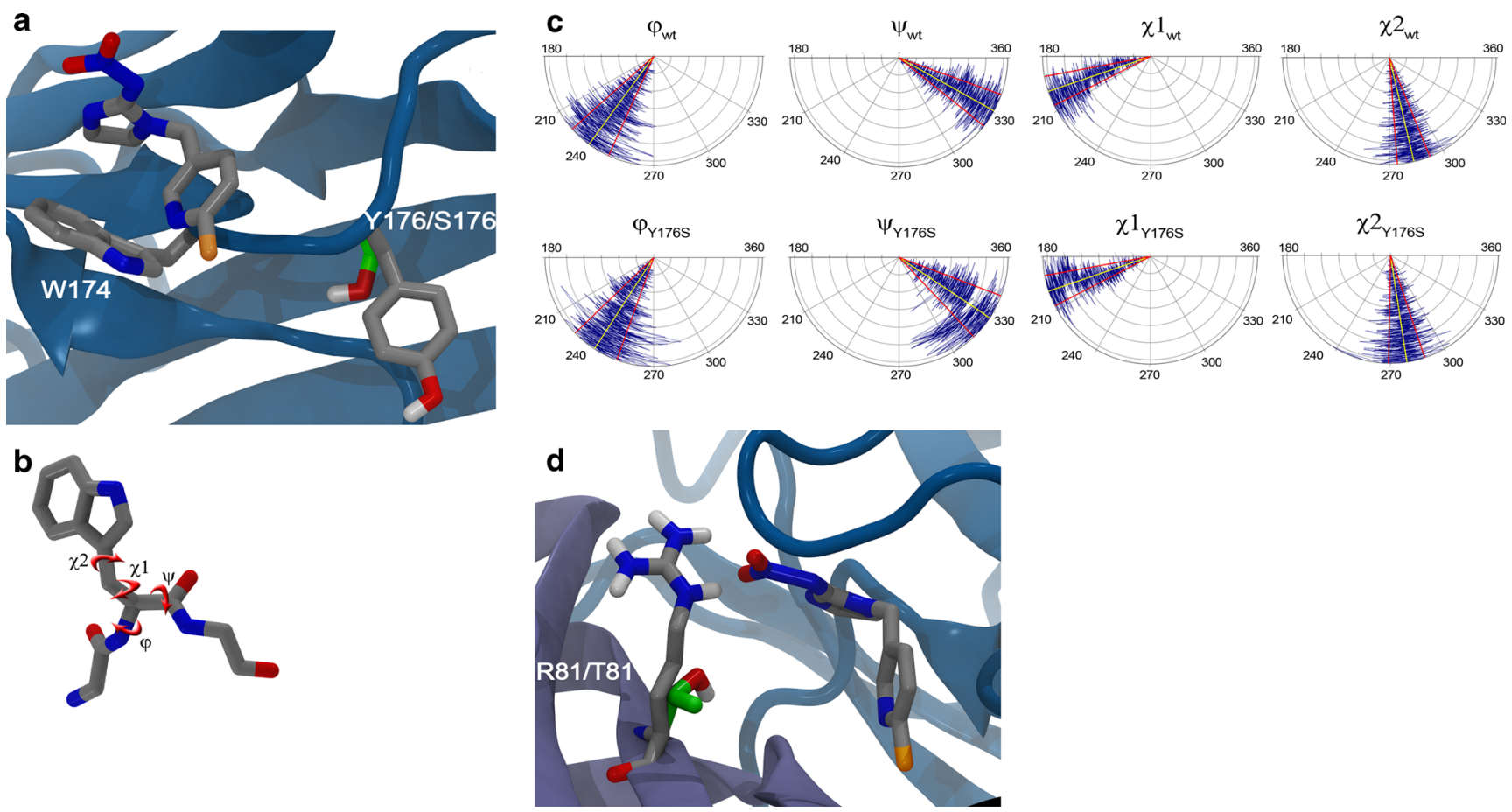

Fig. 1 a Representation of imidacloprid bound at the $\alpha 2 / \beta 1 \mathrm{nAChR}$ interface in Myzus persicae. Mutation of Y176 into serine leads to an increased mobility of the nearby W174. b Supporting information. Depiction of the dihedrals used for the analysis on the tryptophan residue. $\mathbf{c}$ Supporting information. Plots of the mean degree fluctuations of the $\varphi, \psi, \chi^{1}$ and $\chi^{2}$ dihedrals relative to W174 in the wild type (wt) vs. the Y176S mutant along the molecular dynamics runs. Mean values and standard deviations are shown in yellow and red lines, respectively. d R81T mutation causes the loss of the interaction with the nitro group of imidacloprid (mutated residues are shown in green carbons) orchards in South France was found to have a single-point mutation in the loop $\mathrm{D}$ region of the $\mathrm{nAChR} \beta 1$ subunit, which causes an arginine to threonine substitution (R81T) [12] (Fig. 1d). An analogous R81T mutation has also been observed in A. gossypii, although the identification of the mutation in this study occurred after imidacloprid selection pressure for 60 generations in the laboratory environment [30]. Unlike Y151S, the R81T mutation is believed to have a direct effect on the interaction of neonicotinoids within the binding pocket [31]. The presence of this basic amino acid, arginine, being highly conserved across invertebrates but not vertebrates, is believed to be involved in the selectivity of the neonicotinoids to insects. Indeed, site-directed mutagenesis studies on the chick $\alpha 2 \beta 2$ nAChR expressed in Xenopus oocytes demonstrated that mutating the endogenous threonine to an arginine significantly enhanced the responses to imidacloprid [31]. Further molecular dynamics simulations performed in this study showed that, in the native invertebrate system (WT), the positively charged side chain of R81 extends from the $\beta 1$ subunit to interact with the nitro group of imidacloprid (Fig. 1d). In the R81T mutant, the threonine is not as effective as the arginine in its interaction with the ligand owing to its much shorter side chain. As a consequence, the free energy of binding upon mutation of arginine into threonine increases by about $4 \mathrm{kcal} / \mathrm{mol}$ (supporting information), thus indicating a strongly weakened interaction in the mutant receptor. Supporting this hypothesis, radiolabel binding studies on membranes from FRC M. persicae have revealed that the R81T point mutation was associated with the loss of the highest picomolar affinity imidacloprid site, with only a single binding site of low nanomolar affinity present [12]. The loss of the highest affinity binding site explains the observed significant cross-resistance between all commercially available neonicotinoids [3]. The presence of the R81T mutation does not appear to affect the total level of $\mathrm{nAChR}$ expression in aphids as determined by saturation binding experiments [32] nor is there any known fitness penalty.

The identification and detailed characterization of nAChR point mutations associated with neonicotinoid insensitivity has facilitated understanding of the invertebrate receptor. Although being relatively slow to evolve in field populations, the combined impact of generics and the anticipated launch of new-to-market insecticides with a neonicotinoid mode of action [1] is only going to lead to even greater target-site pressure in hemipteran insects. At least in the case of the R81T mutation in $M$. persicae, this is now found throughout southern France and northern Spain [33]. To be truly effective in the field and provide new solutions to growers, novel nAChR modulators are required to overcome both metabolic and target-site resistance to the neonicotinoids. 


\section{References}

1. Jeschke P, Nauen R, Beck ME (2013) Nicotinic acetylcholine receptor agonists: a milestone for modern crop protection. Angew Chem Int Ed 52:9464-9485. doi:10.1002/anie.201302550

2. Gerwick BC, Sparks TC (2014) Natural products for pest control: an analysis of their role, value and future. Pest Manag Sci 70: 1169-1185. doi:10.1002/ps.3744

3. Cutler P et al (2013) Investigating the mode of action of sulfoxaflor: a fourth-generation neonicotinoid. Pest Manag Sci 69:607-619. doi: $10.1002 /$ ps.3413

4. Jeschke P, Nauen R, Schindler M, Elbert A (2011) Overview of the status and global strategy for neonicotinoids. J Agric Food Chem 59: 2897-2908. doi:10.1021/jf101303g

5. Corringer PJ, Le NN, Changeux JP (2000) Nicotinic receptors at the amino acid level. Annu Rev Pharmacol Toxicol 40:431-458

6. Matsuda K, Kanaoka S, Akamatsu M, Sattelle DB (2009) Diverse actions and target-site selectivity of neonicotinoids: structural insights. Mol Pharmacol 76:1-10

7. Jones AK, Brown LA, Sattelle DB (2007) Insect nicotinic acetylcholine receptor gene families: from genetic model organism to vector, pest and beneficial species. Invertebr Neurosci 7:67-73

8. Salgado VL, Saar R (2004) Desensitizing and non-desensitizing subtypes of alpha-bungarotoxin-sensitive nicotinic acetylcholine receptors in cockroach neurons. J Insect Physiol 50:867-879

9. Lind RJ, Clough MS, Reynolds SE, Earley FGP (1998) [3H] Imidacloprid labels high- and low-affinity nicotinic acetylcholine receptor-like binding sites in the aphid Myzus persicae (Hemiptera: Aphididae). Pestic Biochem Physiol 62:3-14

10. Xu X, Bao H, Shao X, Zhang Y, Yao X, Liu Z, Li Z (2009) Pharmacological characterization of cis-nitromethylene neonicotinoids in relation to imidacloprid binding sites in the brown planthopper, Nilaparvata lugens. Insect Mol Biol 19:1-8

11. Collins T, Lansdell SJ, Millar NS (2009) Characterization of insect nicotinic receptors by heterologous expression: insecticide selectivity and the influence of molecular chaperones. Biochem Pharmacol 78:902

12. Bass $\mathrm{C}$ et al (2011) Mutation of a nicotinic acetylcholine receptor beta subunit is associated with resistance to neonicotinoid insecticides in the aphid Myzus persicae. BMC Neurosci 12:51

13. Liu Z, Williamson MS, Lansdell SJ, Denholm I, Han Z, Millar NS (2005) A nicotinic acetylcholine receptor mutation conferring targetsite resistance to imidacloprid in Nilaparvata lugens (brown planthopper). Proc Natl Acad Sci U S A 102:8420-8425

14. Karunker I et al (2008) Over-expression of cytochrome P450 CYP6CM1 is associated with high resistance to imidacloprid in the B and Q biotypes of Bemisia tabaci (Hemiptera: Aleyrodidae). Insect Biochem Mol Biol 38:634-644

15. Jones $\mathrm{CM}$ et al (2011) Age-specific expression of a P450 monooxygenase (CYP6CM1) correlates with neonicotinoid resistance in Bemisia tabaci. Pestic Biochem Physiol 101:53-58

16. Yang $\mathrm{N}$ et al (2013) Transcriptome profiling of the whitefly Bemisia tabaci reveals stage-specific gene expression signatures for thiamethoxam resistance. Insect Mol Biol 22:485-496. doi:10.1111/ imb. 12038

17. Karatolos N, Denholm I, Williamson M, Nauen R, Gorman K (2010) Incidence and characterisation of resistance to neonicotinoid insecticides and pymetrozine in the greenhouse whitefly, Trialeurodes vaporariorum Westwood (Hemiptera: Aleyrodidae). Pest Manag Sci 66:1304-1307

18. Bass C et al (2011) Overexpression of a cytochrome P450 monooxygenase, CYP6ER1, is associated with resistance to imidacloprid in the brown planthopper, Nilaparvata Lugens. Insect Mol Biol 20:763-773. doi:10.1111/j.1365-2583.2011.01105.x

19. Puinean AM et al. (2010) Amplification of a cytochrome P450 gene is associated with resistance to neonicotinoid insecticides in the aphid Myzus persicae. PLoS Genetics 6: doi:10.1371/journal.pgen. 1000999

20. Zhao JZ, Bishop BA, Grafius EJ (2000) Inheritance and synergism of resistance to imidacloprid in the Colorado potato beetle (Coleoptera: Chrysomelidae). J Econ Entomol 93:1508-1514

21. Kshirsagar SDSNS, Moharil MP (2012) Monitoring of insecticide resistance in cotton leafhoppers, Amrasca bigutulla bigutulla (Ishida) Ann Pl Prot Sci 20:3

22. Tiwari S, Mann RS, Rogers ME, Stelinski LL (2011) Insecticide resistance in field populations of Asian citrus psyllid in Florida. Pest Manag Sci 67:1258-1268. doi:10.1002/ps.2181

23. Wang KY, Liu TX, Yu CH, Jiang XY, Yi MQ (2002) Resistance of Aphis gossypii (Homoptera: Aphididae) to fenvalerate and imidacloprid and activities of detoxification enzymes on cotton and cucumber. J Econ Entomol 95:407-413

24. Liu $\mathrm{Z}$ et al (2009) Heteromeric co-assembly of two insect nicotinic acetylcholine receptor alpha subunits: influence on sensitivity to neonicotinoid insecticides. J Neurochem 108:498-506

25. Liu Z, Williamson MS, Lansdell SJ, Han Z, Denholm I, Millar NS (2006) A nicotinic acetylcholine receptor mutation (Y151S) causes reduced agonist potency to a range of neonicotinoid insecticides. J Neurochem 99:1273-1281

26. Yixi $\mathrm{Z}$ et al (2009) Functional co-expression of two insect nicotinic receptor subunits (Nlalpha3 and Nlalpha8) reveals the effects of a resistance-associated mutation (Nlalpha3) on neonicotinoid insecticides. J Neurochem 110:1855-1862

27. Liu Z, Han Z (2006) Fitness costs of laboratory-selected imidacloprid resistance in the brown planthopper, Nilaparvata lugens. Stal Pest Manag Sci 62:279-282

28. Levitt M (2001) The birth of computational structural biology. Nat Struct Mol Biol 8:392-393

29. Ceron-Carrasco JP, Jacquemin D, Graton J, Thany S, Le Questel J-Y (2013) New insights on the molecular recognition of imidacloprid with aplysia californica AChBP: a computational study. J Phys Chem B 117:3944-3953. doi:10.1021/jp310242n

30. Shi X-G, Zhu Y-K, Xia X-M, Qiao K, Wang H-Y, Wang K-Y (2012) The mutation in nicotinic acetylcholine receptor $\beta 1$ subunit may confer resistance to imidacloprid in Aphis gossypii (Glover). J Food Agric Environ 10:1227-1230

31. Shimomura M, Yokota M, Ihara M, Akamatsu M, Sattelle DB, Matsuda K (2006) Role in the selectivity of neonicotinoids of insect-specific basic residues in loop D of the nicotinic acetylcholine receptor agonist binding site. Mol Pharmacol 70:1255-1263

32. Beckingham C, Phillips J, Gill M, Crossthwaite AJ (2013) Investigating nicotinic acetylcholine receptor expression in neonicotinoid resistant Myzus persicae. FRC Pestic Biochem Physiol 107:293-298

33. Slater R, Paul VL, Andrews M, Garbay M, Camblin P (2012) Identifying the presence of neonicotinoidresistant peach-potato aphid (Myzus persicae) in the peach-growing regions of Southern France and Northern Spain. Pest Manag Sci 68:634-638 